\title{
Siberian Snakes and Future Polarized Beams
}

\author{
THOMAS ROSER \\ Randall Laboratory of Physics \\ The University of Michigan \\ Ann Arbor, MI 48109-1120 U.S.A.
}

As you probably all know, the spin vector rotates in a magnetic field by an angle that is $\mathrm{G} \gamma$ times larger than the rotation angle of the momentum. $\mathrm{G}$ is the anomalous magnetic moment of the proton and $\gamma$ is the relativistic energy factor. Thus in a circular accelerator with a vertical holding field the spin precesses around the vertical field. The number of precessions for every revolution is called the spin tune in analogy to the betatron tune, which is the number of transverse oscillations for every revolution. As long as the spin points in the vertical direction, it simply precesses around its own axis. However, if in addition horizontal fields are present depolarizing resonances will occur. These horizontal fields kick the spin away from the vertical direction and if all these kicks add up coherently rapid depolarization will occur.

There are two kinds of depolarizing resonances. Imperfection depolarizing resonances are caused by horizontal fields that are produced by magnet misalignments. If the particle sees the kicks with a frequency that corresponds exactly to the spin tune, coherent build-up occurs. Thus, the resonance occurs when the spin tune is equal to an integer. Since the spin tune increases with the energy, an imperfection resonance is crossed about every $0.6 \mathrm{GeV}$. There are about 40 imperfection resonances at the AGS and some 35,000 at the SSC. Clearly these resonances are very numerous and each one of them has the potential to depolarize the beam. The second type of depolarizing resonances are called intrinsic resonances. They are caused by the horizontal field needed for vertical focusing. In this case, the resonance condition is satisfied when the spin tune is equal to the vertical betatron tune plus a multiple of the superiodicity. This condition results from fact that the frequency with which the particles see horizontal fields depends both on their vertical oscillation and the distribution of the quadrupoles. With a superiodicity of 12 and a vertical betatron tune $\nu_{\mathrm{y}}$ of 8.75 there are only 6 intrinsic resonances at the $\mathrm{AGS}^{[1]}$. At all accelerators there are only a few intrinsic resonances. However, they are generally much stronger than the imperfection resonances.

What can we do to avoid depolarization from such resonances? First of all one can reduce the magnitude of the the horizontal driving fields. For example, the magnet misalignments can be corrected using steering magnets. The 
resulting corrected orbit is then basically flat. Alternatively, one can precisely survey the ring magnets which also reduces the strength of the imperfections. A similar procedure for the intrinsic resonance would consist of reducing the amplitude of the betatron oscillations by reducing the beam emittance.

A second approach to avoid depolarization is reducing the number of coherent kicks. A typical small horizontal field produces a very small kick, but every time the particle goes around it sees the same kick and the resulting coherent build-up will produce depolarization. By going through the resonance condition very rapidly, this coherent build up can be avoided. This technique was successfully used at the ZGS, the AGS and KEK. By changing the betatron tune rapidly with fast, pulsed quadrupoles the resonance condition was crossed very fast. Using these techniques, 39 imperfection resonances and 6 intrinsic resonances were crossed to reach the energy of $22 \mathrm{GeV}$ at the AGS. As I mentioned before, the situation would be much more challenging at the SSC.

A very different and elegant scheme to overcome depolarizing resonances was proposed by Derbenev and Kondratenko from Novosibirsk ${ }^{[2]}$. They proposed to include a $180^{\circ}$ spin rotator in an accelerator. Let me illustrate the principle of this scheme with a ring that has two of these spin rotators. One of them rotates the spin around the longitudinal direction and the other around the radial direction. As a result the energy dependent spin precession around the vertical direction in the first half of the ring gets reversed in the second half. Thus the spin tune of the ring is not energy dependent any more. Since both horizontal polarization components are reversed every revolution, the spin-tune is in fact $1 / 2$. This means every kick in one turn is exactly canceled during the next turn. This brilliant trick stabilizes the spin direction. For this particular ring the stable polarization direction is vertically up in one half and down in the other half. Such $180^{\circ}$ spin rotations are now commonly named 'Siberian Snakes' after their place of invention and the wiggler type magnet structure that was first proposed as an implementation. We thought this to be such a good idea, that we decided to test it experimentally at the Indiana University Cyclotron Facility (IUCF) where commissioning of a small synchrotron storage ring was just starting. It was intended to be used for nuclear physics experiments with internal targets, and it had long straight sections for this purpose. This ring was ideally suited for the installation of a Siberian Snake, since a $180^{\circ}$ spin rotator that does not effect the trajectory of the particle requires a considerable amount of space. Fig. 1 shows the IUCF ring with the spin rotator installed in one of the straight sections. The spin rotator consists of a solenoid that rotates the spin around the longitudinal direction and of 4 quadrupoles on either side to compensate for the orbit focusing and coupling that is introduced by the solenoid. With only one spin rotator, unlike in the example mentioned before, the stable spin direction is now in the horizontal plane. Looking from above, the arrows in Fig. 1 show the stable spin direction at different places around 
the ring. These directions will change with the energy; however, the spin tune remains $1 / 2$ and energy independent.

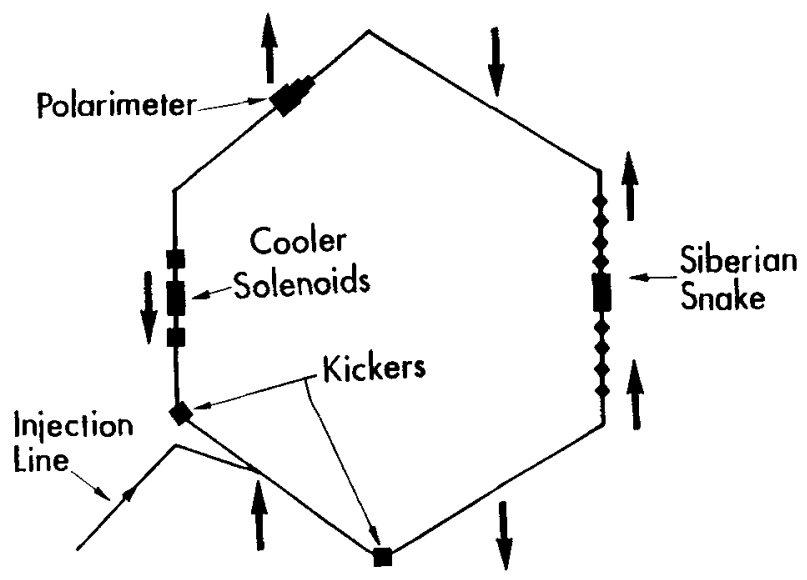

Fig. 1. The IUCF Cooler Ring with the Siberian Snake.

An experimental test of the Siberian Snake concept consists of first establishing the presence of depolarizing resonances and then demonstrating the restoration of full polarization with the Snake. Ernest Courant calculated the strength of the depolarizing resonances of the IUCF ring and indeed there are two resonances in the energy range of the IUCF Cooler ring: one imperfection resonance at $\mathrm{G} \gamma=2$ and one intrinsic resonance at $\mathrm{G} \gamma=-3+\nu_{\mathrm{y}}$.

About half a year ago we started measurements with the Siberian Snake. Fig. 2 shows the first recently published results. ${ }^{[3]}$ The measured polarization is shown as a function of a longitudinal imperfection field generated by small solenoids which were located just opposite to the Snake. The longitudinal field determined the strength of the imperfection resonance. The energy was kept fixed at $104 \mathrm{MeV}$, which is just below the resonances energy of $108 \mathrm{MeV}$ corresponding to $\mathrm{G} \gamma=2$. We found that without the Siberian Snake we got a very sharp peak reaching full polarization only if the longitudinal imperfection field is exactly set to zero. However, when we turned the Snake on, all the structure completely disappears and we measured constant polarization independent of the imperfection field. We took this as the first indication that the Siberian Snake concept works. The peak obtained without the Snake should be predictable by calculating the combined spin-precession of the longitudinal imperfection field and the ring dipole magnets. The curve shown in Fig. 2, however, is not very accurate; presumably we were too close to the resonance to make a good prediction. 


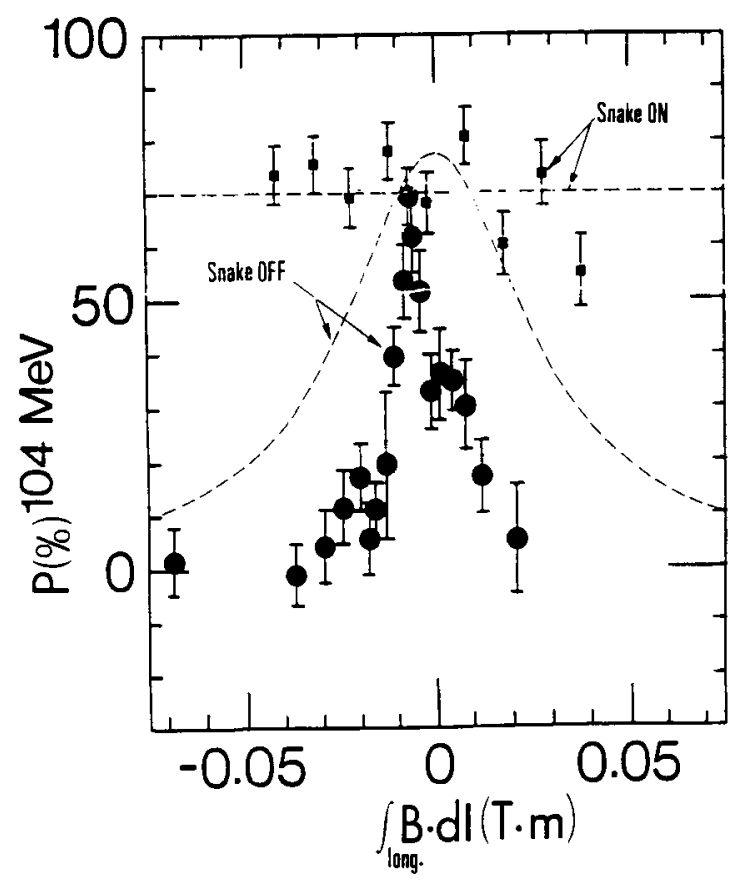

Fig. 2. The Siberian Snake overcoming the effect of the $\mathrm{G} \gamma=2$ imperfection resonance.

For our tests at IUCF we are using a solenoid to produce the spin rotation. To obtain a constant spin rotation, the strength of the solenoid has to be scaled with the momentum. A solenoid spin rotator becomes rather impractical at AGS energies and impossible at even higher energies. Already early on, several people suggested the use of a very different magnet structure at high energies. It consists of a sequence of horizontal and vertical dipole magnets, each of which rotates the spin by a multiple of $45^{\circ}$. Adding up all these spin rotations, while properly taking into account that spin rotations do not commute, results in a spin rotation of $180^{\circ}$ around a horizontal direction. The advantage of using a transverse field to obtain the spin rotation is that for relativistic particles the required $\int \mathrm{B} \cdot \mathrm{d} \ell$ is independent of energy: the same Siberian Snake will work at moderate energies and at SSC energies.

The protons will make excursions inside such a Siberian Snake. At $1 \mathrm{TeV}$ the amplitude of these excursions is about $1.5 \mathrm{~mm}$, but at lower energies the excursions are much bigger. In fact at very low energy, the large excursions make the construction of a Snake with transverse fields infeasible. This was one of the reasons why we used a solenoid at IUCF. For intermediate energy machines, such as the AGS, both types of Snake constructions are impractical. However, a partially excited Siberian Snake could provide an alternative in this energy range where depolarizing resonances are relatively weak. ${ }^{[4]}$ 
When I first told Kent about the idea of using a partially excited Snake, I was still very unsure whether a partial Snake could be useful at all. It was very important to me at that time that I received support and encouragement from Kent to pursue this idea further. Fig. 3 shows the calculated polarization after tracking through an imperfection resonance. It shows that a $5 \%$ partial Snake will trigger complete spin-flip as long as the resonance is relatively weak. Only for a resonance strength of about 0.03 the imperfection resonances and the partial Snake start to interfere and the beam will depolarize. Since at the AGS all imperfection resonance strengths are below this 0.03 level, such a $5 \%$ partial Snake would eliminate the need for the present complex correction schemes. Partial Snakes could be very useful in intermediate energy machines like the AGS, the TRIUMF KAON, or boosters at Fermilab and SSC.

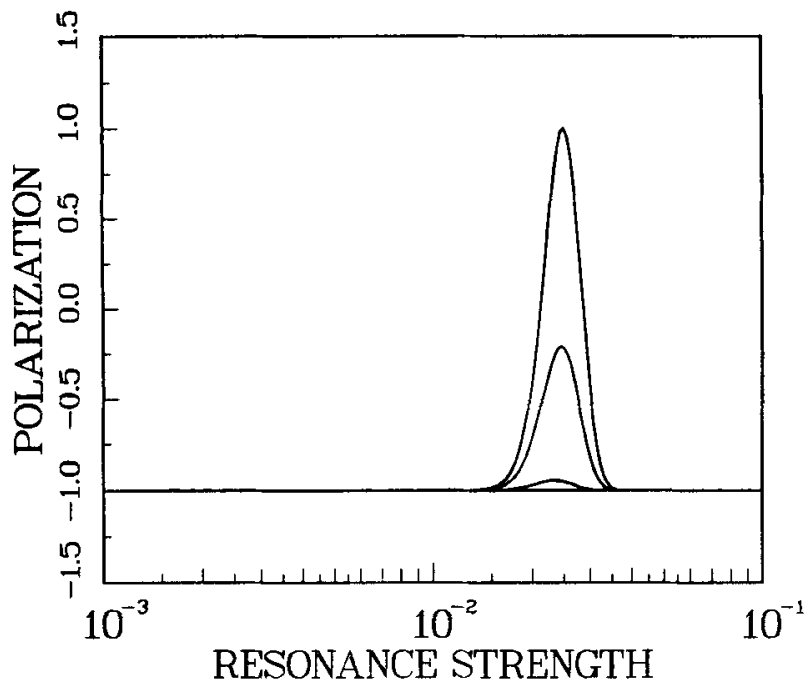

Fig. 3. Calculated polarization after accelerating through an imperfection resonance with a 5\% partially excited Siberian Snake. Each curve corresponds to a different relative phase angle between the Snake and the resonance.

Since the partial Snakes seem to work for weak resonances but a full Snake is required for stronger resonances, there must be some kind of relation between the required Snake and the resonance strength. In a simple picture the spin rotation of the Snake should be larger than the spin rotation from the horizontal fields that drive the resonance. With a partial Snake that rotates the spin by an angle $\delta$ that is smaller than $180^{\circ}$, resonances with a strength of less than $\frac{\delta}{2 \pi}$ can be tolerated. With one Snake the strength has to be less than 1/2, with two Snakes it has to be less than 1 and so on. Although these are somewhat naive limits, we are fairly confident that this estimate is correct up to a resonance strength of about 1 , which corresponds to an energy of about $500 \mathrm{GeV}$. 
At even higher energies two new problems appear. One of them was to my knowledge first pointed out by Kent at the Siberian Snake Workshop 1988 in Minnesota. He pointed out that multiple Snakes may not increase the tolerance for imperfection resonances which are directly driven by random error fields. This type of imperfection resonances are called weak or background resonances. Also, the naive limit is probably not correct for strong, overlapping resonances.
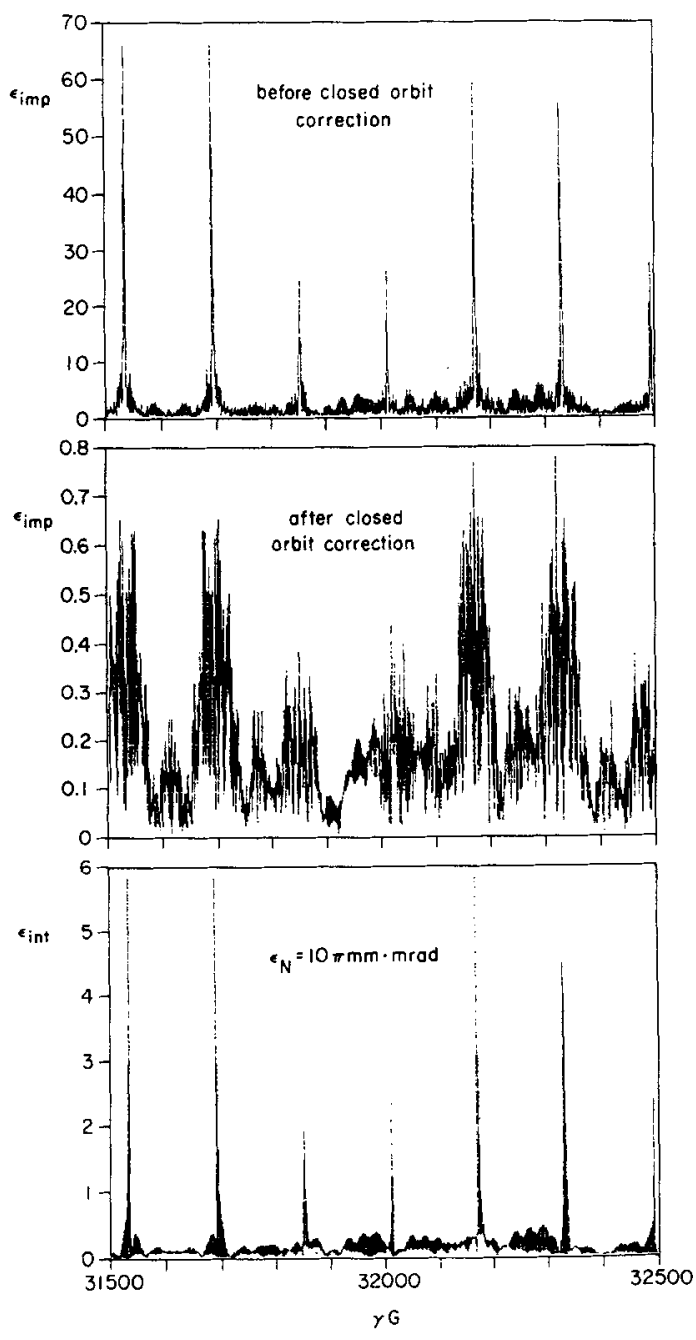

Fig. 4. The imperfection resonance strengths calculated for the SSC lattice (from Ref. 5).
Let me talk a little more about these weak resonances. By studying the strength of imperfection resonances at the AGS, Kent developed a very simple and nice model. The vertical motion in an accelerator behaves like an oscillator and can be driven by small imperfection fields. The amplitude depends on how close the frequency of the driving fields is to the resonance frequency, which is the betatron tune. For well matched frequencies the resulting large vertical excursions lead to large horizontal fields in the quadrupoles and strong depolarizing resonances. ${ }^{[5]}$ On the other hand far away from the vertical betatron tune, the horizontal error fields are not capable of generating large vertical amplitudes. In this case, depolarization comes directly from the misalignment field. At AGS energies these imperfection resonances are very weak and can easily be handled by a Siberian Snake. However, at SSC energies the situation is very different. With the kind permission of the authors, S. Y. Lee and E. D. Courant, ${ }^{[6]} \mathrm{I}$ am showing in Fig. 4 a calculation of the strengths of depolarizing resonances at the SSC. 
The top picture shows the strength of imperfection resonances for random misalignments. You can see a rather low background level and large peaks at the location of strong intrinsic resonances. The strong imperfection resonances can be treated quite effectively by correcting the closed orbit. With residual closed orbit amplitudes of less than $0.3 \mathrm{~mm}$, all these peaks are reduced by two orders of magnitude and all imperfection strengths are well below a strength of one. However, the weak imperfection resonances are not effected at all. I remember Kent making the same prediction a year ago: a quadrupole misalignment of 0.1 $\mathrm{mm}$ would lead to weak imperfection resonances with a strength of about 0.2. Notice that 0.2 is still smaller than one. However, with a rms value of 0.2 , some of the 30,000 weak imperfection resonances are likely to have a strength bigger than one. This is a potential problem for which I don't think a solution has been found yet.

The second problem I was mentioning before was dealt with very nicely by S. Y. Lee and E. D. Courant in a simulation that tracks particles through a set of strongly overlapping intrinsic and imperfection resonances. ${ }^{\left[{ }^{[6]}\right.}$ They determined the limiting resonance strengths for which polarization is still preserved; they found that these limits scale with the number of Snakes as expected for intrinsic resonances and strong imperfection resonances. With 26 Snakes in the SSC the intrinsic resonance strength would have to be smaller than 5.2 and the strength of the strong imperfection resonances would have to be smaller than 2.6. Fig. 4 shows that both conditions are easily met for the SSC lattice. On this very positive note, I would like to end my talk. I am sure that Kent would have liked to see such a result.

This work was supported by the U. S. Department of Energy. 


\section{REFERENCES}

1. F. Z. Khiari et al., Phys. Rev. D39, 45 (1989).

2. Ya. S. Derbenev and A. M. Kondratenko, Part. Accel. 8 , 115 (1978).

3. A. D. Krisch et al., Phys. Rev. Lett. $\underline{63}, 1137$ (1989).

4. T. Roser in Proceedings of the $8^{\text {th }}$ Int. High Energy Spin Physics Symposium, Minneapolis 1988, edited by K. Heller (AIP Conf. Proc. 187).

5. K. M. Terwilliger et al., Trans. in Nucl Sci. NS-32, 2635 (1985).

6. S. Y. Lee and E. D. Courant, Phys. Rev. D41, 292 (1990). 